Regards sur l'économie allemande

Bulletin économique du CIRAC

$69 \mid 2004$

Varia

\title{
Influence allemande à Bruxelles : un état des forces économiques
}

Florence Autret

\section{OpenEdition}

\section{Journals}

Édition électronique

URL : http://journals.openedition.org/rea/3283

DOI : $10.4000 /$ rea.3283

ISBN : 978-2-8218-0834-8

ISSN : 1965-0787

\section{Éditeur}

CIRAC

\section{Édition imprimée}

Date de publication : 1 décembre 2004

Pagination : 11-16

ISSN : 1156-8992

\section{Référence électronique}

Florence Autret, «Influence allemande à Bruxelles : un état des forces économiques », Regards sur l'économie allemande [En ligne], 69 | décembre 2004, mis en ligne le 27 janvier 2009, consulté le 02 mai 2019. URL : http://journals.openedition.org/rea/3283 ; DOI : 10.4000/rea.3283 


\title{
Influence allemande à Bruxelles : un état des forces économiques
}

\author{
Florence Autret
}

Le débat récent sur la "perte d'influence de la France à Bruxelles " ouvre une perspective dans le traitement des questions européennes. Pour une fois, il n'est plus question de mécano institutionnel mais de pouvoir, au sens strict, c'est-à-dire de la capacité à faire, concrètement, bouger les pièces sur l'échiquier politique et économique. Cette réflexion, encore embryonnaire, peut révolutionner notre manière de voir et de penser l'Europe, en particulier parce qu'elle met en évidence le rôle éminent qu'y jouent les groupes d'intérêt, généralement au vu et au su de tous. Comment, de ce point de vue, l'Allemagne tiret-elle son épingle du jeu ? Et comment sont structurés la présence et les leviers d'action de ses lobbies?

A Bruxelles, la participation des groupes d'intérêt à la conception et à l'élaboration des politiques européennes est plus que tolérée, elle est bienvenue. Au moins deux des trois sommets du triangle institutionnel ont un intérêt stratégique à s'attacher l'expertise d'experts extérieurs. II s'agit du Parlement (où sont représentés les peuples) et de la Commission (à qui revient le rôle de proposer et d'exécuter les politiques européennes), la troisième étant le Conseil des ministres (où sont représentés les Etats). Cette situation n'a rien de surprenant pour les Allemands alors qu'elle est déroutante pour les Français.

Car autant le "lobbying " est tabou en France, autant il constitue en Allemagne, sous le nom de "représentation des intérêts " (Interressenvertretung), un rouage naturel et essentiel de l'économie sociale de marché. L'art. 9 (3) de la Loi fondamentale garantit en effet à tous et dans toutes les professions le droit de fonder des associations pour la sauvegarde et l'amélioration des conditions de travail et des conditions économiques. Ce droit fondamental est à la base de l'autonomie des partenaires sociaux en matière de macro-régulation sociale ; il fonde aussi l'organisation de la représentation des intérêts de la société civile via les corps intermédiaires que sont, dans le domaine économique, les associations professionnelles (Verbände). Regroupées au sein de puissantes fédérations de branche, elles font partie intégrante de cette forme négociée du capitalisme appelée communément le « modèle rhénan » et où l'acteur public lui-même est « partie prenante de la société civile » (Colletis, 2004).

En France, le néocorporatisme de l'ère gaullienne s'est montré d'emblée très ambigu quant à l'autonomie laissée aux acteurs non publics. II a d'ailleurs souvent consisté à absorber la sphère économique dans la sphère politique (cf. la création de cette troisième chambre destinée à assurer la représentation des "forces vives » : le Conseil économique et social). En République fédérale, à l'inverse, les entreprises - via les structures qui en émanent - participent directement et activement à l'action politique et sociale. Intégrées en amont du processus de prise de décision politique, elles agissent au nom de l'intérêt général (Gemeinwohl), faisant entendre légitimement la voix collective d'une branche, d'un secteur, du patronat, du salariat ou d'une religion. Ce néocorporatisme pluraliste qui brouille, aux yeux d'un observateur français, les frontières entre public et privé, est générateur de cette "culture du consensus " qui permet, outre-Rhin, une convergence effective des intérêts entre groupes sociaux.

Lobbying bienvenu à Bruxelles

RFA : un capitalisme négocié qui permet de ...

... concilier les intérêts des divers groupes de la société civile 
Une forte représentation au niveau des partenaires sociaux européens

Mais des syndicats sous-représentés par rapport au patronat

Fédérations sectorielles : le poids de l'industrie allemande

65 bureaux de liaison et représentations propres...
Acteur historique de l'intégration européenne, l'Allemagne a exporté à Bruxelles son mode de fonctionnement. De tous les acteurs de l'influence, les fédérations professionnelles (Verbände) et les syndicats sont de loin les plus importants par leur nombre. Dans ce domaine, la présence allemande s'organise en plusieurs cercles.

Le premier réunit les organisations proprement européennes, où il faut à nouveau distinguer plusieurs niveaux. Tout d'abord, les organisations confédérales ou partenaires sociaux: Unice (confédération patronale européenne), CES (confédération européenne des syndicats) et CEEP (centre européen des entreprises à participation publique et des entreprises d'intérêt économique général) qui jouissent d'un pouvoir quasi-législatif en matière sociale depuis l'entrée en vigueur du traité de Maastricht. Ces organisations rassemblent des confédérations nationales, à savoir : le BDI (industrie) et le BDA (patronat) à l'Unice et le DGB (syndicalisme) à la CES. Au CEEP, ce sont une cinquantaine d'entreprises ou d'organisations représentatives compte tenu de la décentralisation de l'organisation des secteurs de l'eau, de l'électricité, des déchets, des hôpitaux, etc. en Allemagne.

Mais autant les organisations allemandes savent faire entendre leur voix au sein de l'Unice, présidé, du reste, par l'ancien président du directoire de BASF (et toujours président de son conseil de surveillance), Jürgen F. Strube, autant leur influence est plus limitée du côté de la CES. "La CES fonctionne comme une superstructure plutôt que sur un mode fédéral, ce qui met le DGB mal à l'aise et lui retire de son influence, malgré le poids des syndicats allemands ", analyse un spécialiste du dialogue social. A plusieurs reprises, la confédération syndicale (DGB) s'est trouvée isolée lors de discussion sur des projets d'accords qui allaient être adoptés dans le cadre du dialogue social. Le niveau sectoriel, celui auquel s'exprime le mieux la force des syndicats allemands, est peu organisé à l'échelon de l'Europe, à l'exception de la Fédération européenne de la métallurgie (FEM). En 2001, la pondération des voix, établie en fonction du nombre d'adhérents, réservait $28 \%$ des droits de vote à IG Metall (pour mémoire : les syndicats britanniques disposaient de $10 \%$ des voix et les français, de $3 \%$ ). La FEM s'est en effet montrée très en pointe par le passé pour pousser une forme d'européanisation de la négociation collective qui avait été reprise au niveau multisectoriel par la CES dans le cadre de l'accord dit « de Doorn » (voir REA 45/00).

S'ajoutent aux confédérations les fédérations sectorielles européennes qui comptent, pour les plus importantes, jusqu'à 150 collaborateurs branchés en permanence sur la préparation et la discussion de la législation européenne. La présence d'Allemands au sein de ces organisations est à l'image du poids de l'industrie allemande dans ces secteurs et, dans nombre d'entre elles, le président ou le dirigeant est de nationalité allemande. On citera pêle-mêle, pour les présidences, l'European Association of Automotive Suppliers (Jürgen Harnisch, président du directoire de ThyssenKrupp-Automotive), la Fédération de l'industrie européenne de la construction FIEC (Wilhelm Küchler, l'ancien président de la fédération allemande de la construction) et la fédération européenne de l'industrie chimique (Eggert Voscherau, président du directoire de BASF) ou, pour les directions générales, Ulrich Paetzold à la FIEC et Corina Hebestreit chez Euromines.

Le second cercle regroupe les organisations allemandes qui ont choisi, outre leur participation aux organisations européennes, d'avoir une présence propre auprès des institutions européennes. Là encore, la présence allemande est importante. Ces représentations des grandes institutions et organisations allemandes, appelées bureaux de liaison européens (EU Verbindungsbüros) sont au nombre de 65. Parmi elles, le bureau européen du BDI, fort d'une dizaine de permanents (5 seulement pour le BDA installé sur le même palier), se conçoit comme un simple poste d'observation et un intermédiaire entre le patronat alle- 
mand et les décideurs européens. II n'a pas de fonction politique. Comme au Medef, c'est toute l'organisation du BDI, comme du BDA, à Berlin, qui a intégré de façon transversale la dimension européenne à ses travaux. Plusieurs représentations sectorielles sont d'ailleurs installées dans le même immeuble, au 31 rue du Commerce : celle de l'automobile (VDA), de la chimie (VCI) ou des notaires. II n'est pas rare qu'elles soient plus importantes numériquement, comme celle du VDMA, la puissante fédération de la construction mécanique.

Toutefois, au regard du relativement faible espace laissé à Bruxelles aux fédérations professionnelles sectorielles, on peut se demander si, en projetant sur Bruxelles des réflexes nationaux, les Allemands n'ont pas surinvesti. Le rôle de ces organisations connaît deux limites majeures. La première tient au pouvoir européen lui-même, avant tout réglementaire, qui n'offre pas les mêmes possibilités de coopération que le pouvoir fédéral ou celui des Länder allemands. L'autre limite tient à leur taille, qui est un facteur évident de paralysie même lorsqu'elles ont prévu un système de vote à la majorité pour dégager des positions communes. Apparemment toutes puissantes (lorsqu'elles alignent les chiffres impressionnants du nombre cumulé de salariés de leurs membres ou de leur chiffre d'affaires), ces organisations peuvent s'avérer des colosses au pied d'argile. D'où l'intérêt de maintenir une représentation nationale et de diversifier ses relais d'influence.

Un nombre croissant de grandes entreprises allemandes a opté pour une stratégie d'influence non sectorielle qui n'est du reste pas exclusive du rôle tenu dans telle ou telle fédération. Cette démarche ne concerne qu'un nombre limité d'entreprises qui ont choisi d'ouvrir leur propre représentation à Bruxelles (comme elles l'ont souvent fait à Bonn, hier, et à Berlin, aujourd'hui). Elles sont regroupées au sein d'un réseau informel regroupant les chefs de leurs bureaux européens : ce EU Wirtschaftskreis regroupe une trentaine de membres, plus le BDI. II est comparable en tous points au « cercle des représentants permanents français » (qui, au passage, ignore l'existence de son homologue allemand).

Les plus grandes de ces entreprises sont également celles qu'on trouve au conseil d'administration des grands lobbys transnationaux ou parmi les fondateurs des think tanks européens. C'est ainsi que huit des 45 dirigeants membres de l'European Roundtable of Industrialists sont allemands et que, parmi les 42 membres du Transatlantic Business Dialogue (TABD) figurent trois groupes allemands. Plusieurs entreprises, enfin, investissent dans les think tanks qui comptent à Bruxelles: The European Policy Center, Friends of Europe ou encore le CEPS (Center for European Policy Studies) et se trouvent ainsi complètement intégrées dans le paysage bruxellois à l'instar des multinationales françaises, britanniques ou américaines.

\section{European Round Table of Industrialists (ERT) :}

Fondé en 1983 avec une majorité de groupes britanniques et américains, l'ERT est un club de grands patrons plus qu'une organisation professionnelle. Elle a été très active dans la préparation des quelques 300 directives et règlements adoptés pour la réalisation du Marché Unique. Ses travaux portent actuellement sur tous les grands sujets de politique économique de l'Union, comme la compétitivité, l'emploi ou les normes comptables. Membres allemands: Gerhard Cromme, président de l'ERT (ThyssenKrupp); Wulf Bernotat (E.ON); Henning Kagermann (SAP); Heinrich v. Pierer (Siemens); Bernd Pischetsrieder (VW); Kai-Uwe Ricke (Deutsche Telekom); Manfred Schneider (Bayer); Jürgen Weber (Deutsche Lufthansa).

Transatlantic Business Dialogue (TABD) :

Le TABD, qui regroupe quant à lui des entreprises, a été créé à l'initiative de l'ancien ministre fédéral américain au commerce extérieur Ron Brown, avec le soutien en Europe des commissaires britannique Sir Leon Brittan et allemand Martin Bangemann, respectivement en charge du Commerce extérieur et de l'Industrie. Actuellement coprésidé par Niall Fitzgerald (Chairman et CEO Reuters Group PLC) et E. Neville Isdell (Chairman et CEO The Coca-Cola Company), il compte trois membres allemands : les groupes BASF, Deutsche Bank et SAP.

Pour être influent, il ne suffit pas d'être présent. II faut également être entendu par les décideurs ou être investi d'une légitimité à décider et/ou agir. Cette
... au pouvoir cependant limité

Des réseaux informels non sectoriels et ...

... une forte présence des grandes entreprises dans les réseaux multinationaux et les think-tanks

Trois leviers d'influence 
Auprès de la Commission, la carte de l'expertise

Un élément de politique économique

L'impressionnant investissement des Länder

Un rapport de force triangulaire participation est bien difficile à appréhender, et les obligations de publicité faites aux institutions en la matière restent limitées. A cet égard, un important travail théorique reste à faire sur la problématique de la gouvernance. Rappelons cependant que, l'architecture institutionnelle de l'Union reposant sur trois piliers, les leviers de l'influence sont également au nombre de trois, à savoir : la participation au travail de la Commission européenne, l'influence sur les positions défendues au Conseil des ministres et, enfin, la capacité à faire relayer ses positions au moment du débat parlementaire.

Les acteurs économiques allemands ont deux atouts majeurs pour répondre à la demande forte d'expertise de la Commission européenne. Premièrement, elle leur est familière. Autant la tradition française veut que l'expertise soit réputée intégrée à l'administration de l'Etat, autant, en République fédérale, les entreprises et leurs représentants sont constamment sollicités par les autorités publiques à l'appui des politiques de régulation (pour la libéralisation des services de réseaux, par exemple) et des politiques structurelles (formation, emploi). Leur second atout tient à la variété et à l'importance de leur présence à Bruxelles, qui joue un effet démultiplicateur sur le premier. Cet investissement leur permet d'être une force de proposition auprès des services de la Commission et un interlocuteur fiable. Les méthodes de travail de la Commission européenne offrent de multiples occasions de valoriser ces atouts. La « Plateforme européenne pour les technologies de l'hydrogène et des piles à combustibles » (voir REA 66/04) mise en place au début de l'année par la Commission européenne en offre l'exemple. Tous les grands acteurs de la filière sont présents au sein de son comité de pilotage : les industriels et leurs fédérations professionnelles, mais également les acteurs de la politique industrielle et de recherche. Les enjeux d'une telle participation sont multiples : technologiques, industriels et commerciaux, puisqu'elle renforce la position de l'industrie et des centres de recherche allemands dans la filière.

Au niveau du Conseil des ministres, la faculté de faire valoir les positions des acteurs économiques est facilitée par l'approche qu'ont leurs autorités des questions européennes, considérées comme un élément de politique intérieure. En témoignent les modalités de la coordination interministérielle destinée à faire émerger une position nationale, qui diffèrent profondément en France et en Allemagne. Pas de secrétariat général à la coordination interministérielle pour les questions européennes ou SGCl placé directement sous l'autorité du Premier Ministre, outre-Rhin. La préparation des positions allemandes échoit, au sein du gouvernement fédéral, au ministère de l'Economie (sauf pour la justice, les affaires intérieures et la politique étrangère et de sécurité). En cas de divergence avec le gouvernement fédéral, le Parlement, où les lobbys ont traditionnellement une présence forte, fonctionne comme une corde de rappel.

Si les traités ne connaissent que « la République fédérale », le pouvoir fédéral doit, lui, veiller à associer les Länder, en particulier dès lors que ses positions européennes touchent à leurs compétences. Cela vaut notamment pour les aides aux entreprises, sur lesquelles la Commission européenne exerce un contrôle extrêmement scrupuleux. En Allemagne, en effet, la plus grande partie de ces « aides d'Etat » est en réalité allouée par les Länder. Les procédures d'examen sont donc suivies de près par leur représentation, en lien avec les fédérations professionnelles et les entreprises intéressées, comme cela fut par exemple le cas pour la construction navale ou le charbon.

Comme l'explique un représentant de l'industrie, « les Länder viennent défendre à Bruxelles leur identité » menacée par les transferts de compétences à l'échelon européen et le renforcement subséquent du pouvoir fédéral. Leur présence spectaculaire (avec plusieurs dizaines de permanents, la plus importante est sans conteste celle de la Bavière) participe de l'établissement d'un rapport de force triangulaire entre institutions européennes, fédérales et régionales, qui n'interdit cependant pas la coopération. Ainsi, au sujet de la très délicate révi- 
sion des autorisations accordées aux produits chimiques (la fameuse directive $\mathrm{REACH}$ ), des consultations informelles ont déjà commencé entre la représentation permanente, les industriels et les Länder pour suivre la négociation au plus près. Les députés européens ont d'ores et déjà obtenu de la Commission la réalisation d'une étude d'impact en vue de mieux prendre en compte les intérêts industriels.

\section{Harmonisation européenne et défense de l'économie sociale de marché}

L'inspiration libérale de nombreuses propositions de lois de la Commission européenne pose un problème particulier à la République fédérale lorsqu'elle menace directement les fondements de l'économie sociale de marché. Exemple: la société anonyme européenne dont le projet a achoppé pendant des années sur la question de la représentation des salariés dans les organes dirigeants. Le risque était qu'en introduisant dans tous les pays de l'Union une forme de société ne prévoyant pas de participation des salariés, la Mitbestimmung soit contournée, en Allemagne même, grâce au recours au statut de société européenne. Le - très long - travail parlementaire coordonné par le député démocrate-chrétien Winfrid Menrad a permis que la réglementation adoptée exclue ce risque en réintroduisant le facteur national dans les règles de gouvernance de la société européenne. Mais ce compromis n'en est qu'à moitié un, puisqu'il rend factice l'unicité du statut de la société européenne. La création d'un statut unique a donc été sacrifiée sur l'autel de la Mitbestimmung.

Le modèle de l'économie sociale de marché, même s'il est la référence formelle de l'Europe en matière de régulation et qu'il est inscrit comme tel dans les traités, ne fait pas bon ménage avec le mouvement d'harmonisation du continent. Sur de nombreux dossiers, la République fédérale se trouve donc face à un dilemme. Sauvegarder les traits spécifiques du modèle allemand en négociant, au nom de la subsidiarité, des marges de manœuvre nationales fait courir au Standort un risque important en termes de compétitivité. Dès lors, la seule solution est d'exporter le modèle dans les autres pays européens, une tentation qui ressurgit régulièrement.

La problématique des débats qui s'ouvrent sur la $10^{\text {ème }}$ Directive sur les fusions transfrontalières est exactement la même. La question est : comment préserver la Mitbestimmung dans le cadre d'une fusion entre sociétés allemande et française ou allemande et italienne ? Le risque étant, là encore, d'abandonner la cogestion à la faveur d'une fusion transfrontalière - ce que, le cas de la fusion Daimler Chrysler l'a montré, les salariés allemands ne sont pas prêts à accepter.

La position défendue conjointement par le BDI et le BDA est tout à fait éloquente. Les confédérations ne cherchent pas à se servir du levier bruxellois pour limiter la Mitbestimmung en Allemagne. A un compromis prévoyant une dilution - et non une suppression - de la participation des salariés, ils préfèrent l'application du "principe du pays membre d'origine ». On s'oriente ainsi vers une solution où, dans le cas d'une fusion transfrontalière impliquant au moins une société allemande, la parité entre salariés et managers au conseil de surveillance restera la règle, à moins qu'un groupe de négociation ad hoc réunissant les deux parties ne décide d'y déroger spécifiquement.

Tout indique que les parlementaires européens allemands ont exporté à Bruxelles (et à Strasbourg) l'esprit du parlementarisme allemand. Non contents d'être les plus nombreux (la délégation allemande comprend 99 élus), ils y figurent également parmi les plus actifs (avec les Britanniques) et sont réputés pour leur professionnalisme, leur assiduité, mais aussi leur grande proximité avec certains intérêts économiques. Les liens qu'ils entretiennent avec ces derniers peuvent être contractuels ou se nouer au gré des travaux parlementaires. Elmar Brok, par exemple, actuellement président de la Commission des affaires étrangères, précise dans sa déclaration d'intérêts (que les élus ont désormais obligation de rendre publique) qu'il est "senior vice-president for media development » de la Bertelsmann AG.

Mais un élu, même puissant, ne peut rien s'il est isolé, surtout dans l'enceinte parlementaire où les coalitions se font et se défont au gré des textes. II faut agir collectivement. Or la taille de la délégation allemande, ainsi que sa faible dispersion entre groupes, expliquent que ses membres occupent le plus grand nombre de ces postes stratégiques : présidences de commission, coordination de la position d'un groupe politique au sein d'une commission (cette fonction est confiée à un élu dit " coordinateur " pour la durée de la mandature), rapports sur les textes en débat. Ces positions leur permettent de se saisir de sujets stratégiques comme le montrent les exemples de textes examinés au cours de

Le puissant levier du Parlement européen ...

... avec ses présidences de commission 
la dernière mandature (1999-2004) et dont le rapporteur, social-démocrate ou démocrate-chrétien, appartenait à la délégation allemande.

Travaux parlementaires pour lesquels des députés allemands ont été rapporteurs

\begin{tabular}{|ll|}
\hline Texte de la directive / du règlement & Rapporteur \\
- Offre publique d'achat ou d'échange $-13^{\text {ème }}$ Directive (2001) & Klaus-Heiner Lehne (PPE) \\
- Fusions transfrontalières $-10^{\text {eme }}$ Directive (enjeu : participation & \\
des salariés à la gestion) & Klaus-Heiner Lehne (PPE) \\
- Déchets : prévention quantitative et qualitative et recyclage. & Karl-Heinz Florenz (PPE) \\
Communication (2004) & \\
- Environnement et santé : déchets d'équipements électriques et & Karl-Heinz Florenz (PPE) \\
électroniques DEEE (2002 et modif. direct. 2003) & Karl-Heinz Florenz (PPE) \\
- Véhicules hors d'usage. Proposition de directive (2000) & Bernd Lange (PSE) \\
- Qualité de l'air : émissions des véhicules à moteur (2001, 2002) & Bernd Lange (PSE) \\
- Emissions poids lourds & \\
- Contrôle technique routier des véhicules utilitaires circulant dans & Willi Piecyk (PSE) \\
la Communauté & \\
- Programme pluriannuel pour la promotion des sources d'énergie & Werner Langen (PPE) \\
renouvelables (1998-2002). Proposition de décision (1999) & Werner Langen (PPE) \\
- Sidérurgie : code des aides au secteur acier (1999) & Werner Langen (PPE) \\
- Construction navale : la situation dans le monde (2000) & \\
- Assurances : accords, décisions et pratiques concertées, & Werner Langen (PPE) \\
exemption catégorielle (règlement) (2000) & Werner Langen (PPE) \\
- Les services d'intérêt général en Europe (2000) & Etatut de la société coopérative européenne \\
- Statut de la société anonyme européenne & Hans-Peter Mayer (PPE) \\
- Statut de la société anonyme européenne - place des travailleurs & Winfrid Menrad (PPE) \\
\hline
\end{tabular}

Source : www.europarl.eu.int

Les ETATS MEMBres EXPORTENT AU NIVEAU EUROPEen leurs pratiques de l'influence avant de s'adapter, avec plus ou moins de succès, au contexte local. L'Allemagne ne fait pas exception. Elle a conquis une place de premier plan dans le champ de la représentation sectorielle ou quasi-institutionnalisée (partenaires sociaux) et joue un rôle plutôt de suiveur dans les organisations conçues selon les canons britanniques ou américains du lobbying.

Pendant ce temps, en Allemagne, les difficultés économiques, la remise en question des formes de régulation mises en place à partir des années 1950 ainsi que les changements dans la structure du capital des entreprises conduisent à l'éclatement et à la démultiplication des formes de représentation collective (souvent au détriment de l'intérêt général). Dans ce contexte, être présent à Bruxelles comporte un double enjeu. L'espace politique européen, où prédominent le pluralisme et la concurrence dans les modes de représentation, constitue tout d'abord un formidable terrain d'expérimentation pour de nouvelles formes de coopération entre sphères publique et privée. Mais il est aussi un champ à investir d'urgence pour adapter les régulations traditionnelles aux défis lancés par une concurrence internationale exacerbée. Les Allemands l'ont compris, comme le montre la mobilisation de leurs capacités d'influence pour y défendre le modèle de l'économie sociale de marché.

\section{Indications bibliographiques}

AUTRET F., "Coordination syndicale européenne. Premières expériences entre syndicats allemands, belges et néerlandais », Regards sur l'économie allemande, $n^{\circ} 45 / 00$

PaUtrat M.-H., "Lobbying en crise : la fin de l'intérêt collectif ? ", Regards sur l'économie allemande, $\mathrm{n}^{\circ} 60 / 03$

ColletIS G., " Mutation du 'modèle rhénan' et avenir du modèle européen », Regards sur l'économie allemande, $\mathrm{n}^{\circ}$ 67/04

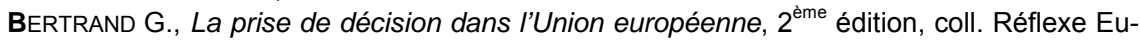
rope, La Documentation Française, 2002

Schumacher, A., « Néocorporatisme et économie sociale de marché », in Commun P. (dir.), L'ordolibéralisme allemand, aux sources de l'économie sociale de marché, Cirac / CICC, 2003 